\title{
HumaNities \\ Psoriasis: \\ Advocating for psychosocial support
}

Aryan Riahi, BSc

Department of Anesthesia, Perioperative Medicine \& Pain Management, Halifax, NS, Canada

$\mathrm{P}$ soriasis is a chronic inflammatory skin condition that is heavily affected by psychosocial factors. Surveys administered by the National Psoriasis Foundation (NPF) report that psoriasis affects enjoyment of life and overall emotional well-being of $82 \%$ and $88 \%$ of patients, respectively ${ }^{1}$.

Medical treatment is inadequate in relieving the psychosocial burden of psoriasis. The majority of patients continue to feel decreased quality of life despite receiving treatment ${ }^{2}$. An emerging ideology of healthcare is the psychosocial model, which considers the patient beyond their physical signs and symptoms. Therefore, psychosocial therapies may play a role in the management of psoriasis.

In this commentary, the psychosocial challenges that patients with psoriasis face will be explored. The multifaceted impacts of psoriasis on quality of life and emerging psychosocial treatments will be the focus of the commentary.

\section{Battling Social Stigma}

Societal stigma and misconceptions are major contributors to the psychosocial burden of psoriasis. In a survey of 1005 people between the ages of 16-64, $50 \%$ of respondents showed discrimination towards psoriatic patients such as reluctance in shaking hands and partaking in sexual relations ${ }^{2}$. Such stigma undermines self-esteem ${ }^{3}$. Patients with psoriasis often report a lack of control and hopelessness in coping with their disease ${ }^{4}$.

Psoriasis hinders social functioning. In one study, 5,604 patients completed surveys about the effects of psoriasis on their quality of life between 2003 to 2011 . When asked , 92\% percent of patients with psoriasis in surveys administered by the National Psoriasis Foundation (NPF) listed their condition as a reason for unemployment ${ }^{1}$. The majority of patients with psoriasis report distress and a lack of efficiency at work ${ }^{2}$. Chronic skin conditions such as psoriasis can lead to a vicious cycle of occupational dysfunction, which leads to lower earnings and a lack of advancement. The resulting distress may then worsen the psychosocial burden of the disease ${ }^{2}$.

\section{Longstanding implications}

The psychosocial impacts of psoriasis are manifold. Patients with psoriasis experience distress similar to that experienced in chronic conditions such as diabetes, cancer, and cardiovascular disease ${ }^{2}$.

Mental health is affected in patients with psoriasis. In a study of 469,097 patients with psoriasis, the third most prevalent comorbidity was depression ${ }^{5}$. Longitudinal studies suggest that antidepressant medications are prescribed twice as frequently in patients with psoriasis (17.8\%) compared to controls $(7.9 \%)^{6}$.

Interpersonal conflict from psoriasis may stem from societal stigma and isolation. Patients with psoriasis often cite feeling self-conscious and shameful which may impede developing meaningful relationships ${ }^{7}$. The psychosocial burden affects family members as well. Family members of patients with psoriasis experience emotional distress (98\%) and worry about the future of the patient $(57 \%)^{8}$.

Psoriasis affects sexual function both psychologically and physically. In a survey of 3485 patients with skin conditions, $34 \%$ of patients with psoriasis reported that their condition interferes with sexual function 9 . Sexual impairment was found to be strongly correlated with depression, anxiety disorders, and suicidal ideation ${ }^{9}$.

The psychosocial burden of disease is not only mentally distressing to patients with psoriasis, but it oftentimes precedes flaring up of the disease ${ }^{10}$. With the severe impediment it imposes on quality of life, treating the psychosocial consequences of psoriasis is pivotal in providing patient-centered care. This is why healthcare providers must complement traditional pharmacologic options with psychosocial therapies.

\section{Beyond Pharmacologic Treatments}

Managing the psychosocial aspects of psoriasis has recently gained momentum in the literature. Kolli et al. suggests an approach that encompasses all four psychosocial dimensions of psoriasis: psychiatric comorbidities, occupational function, relationships, and sexual function ${ }^{2}$. This approach supplements pharmacologic therapies with alternative treatments. For example, psychiatric comorbidities such as depression warrant psychiatric referrals and cognitive behavior therapy. Interpersonal relationships can be improved with referral to support groups.

The initial studies look promising. A 2018 systematic review on psychosocial interventions for psoriasis found that current interventions for patients utilize cognitive behavioral therapy (CBT), mindfulness, meditation, and emotional writing ${ }^{2}$. These techniques were found to significantly increase health-related 
quality of life and decrease patient reported outcomes. However, the evidence is not conclusive. Many studies reporting positive outcomes with psychosocial therapies for psoriasis have a small sample size which results in a low power. The average sample size of the papers included in one recent systemic review was only $81.6^{2}$. While the results are promising, more rigorous and large-scale studies are needed before incorporating these therapies into clinical guidelines.

Social stigma directed towards psoriasis can be addressed with public psoriasis awareness programs. By changing social perceptions and false beliefs towards psoriasis, patients may experience less fear of social rejection and isolation. High school programs run by the Acne and Rosacea Society of Canada offer high school students presentations that educate the population about acne and ways to improve self-esteem for patients suffering from the common disease ${ }^{11}$. These programs can serve a dual purpose of promoting public awareness and effective self-management for patients. Unfortunately, these programs do not include psoriasis. Similar initiatives should be advocated for patients suffering from psoriasis. An improvement in mental health may go a long way in decreasing flare ups and improving quality of life for patients.

The wave of holistic approaches for managing chronic skin conditions has already begun. There are centres for patients with atopic dermatitis where families and patients participate in educational programs. These centers are run by a multidisciplinary team of dermatologists, nurse practitioners, and psychologists who address the unique needs of each patient. Families are also able to partake in support groups and share their experiences with other families. These programs have successfully been implemented across the United States, Europe, and Asia ${ }^{12,13}$. Sadly, similar large scale efforts have not yet been made for psoriasis. With such promising results, it is time for healthcare providers to advocate for similar programs for psoriasis.

\section{Summing up}

The psychosocial burden of psoriasis requires that healthcare professionals take a holistic approach when caring for patients. Patients are more than organ systems. The bigger picture of patient care includes physical, emotional, social, and spiritual well-being. If we want patients with psoriasis to receive well-rounded care, then we must advocate for interventions that promote psychosocial support.

\section{References}

1. Armstrong AW, Schupp C, Wu J, Bebo B. Quality of life and work productivity impairment among psoriasis patients: findings from the National Psoriasis Foundation survey data 2003-2011. PLoS ONE 2012;7(12):e52935.

2. Kolli SS, Amin SD, Pona A, Cline A, Feldman SR. Psychosocial impact of psoriasis: a review for dermatology residents. Cutis 2018 Nov;102(5S):21-25.

3. Łakuta P, Przybyła-Basista H. Toward a better understanding of social anxiety and depression in psoriasis patients: The role of determinants, mediators, and moderators. J Psychosom Res 2017 03;94:32-38.

4. Vardy D, Besser A, Amir M, Gesthalter B, Biton A, Buskila D. Experiences of stigmatization play a role in mediating the impact of disease severity on quality of life in psoriasis patients. Br J Dermatol 2002 Oct;147(4):736-742.

5. Shah K, Mellars L, Changolkar A, Feldman SR. Real-world burden of comorbidities in US patients with psoriasis. J Am Acad Dermatol 2017 Aug;77(2):292.e4.

6. Dowlatshahi, E., Wakkee, M., Herings, R., Hollestein, L., \& Nijsten, T. (2013). Increased Antidepressant Drug Exposure in Psoriasis Patients: A Longitudinal Population-based Cohort Study. Acta Dermato Venereologica, 93(5), 544-550. doi: 10.2340/00015555-1566

7. Feldman SR, Malakouti M, Koo JY. Social impact of the burden of psoriasis: effects on patients and practice. Dermatol Online J 2014 Aug 17;20(8).

8. Eghlileb AM, Davies EEG, Finlay AY. Psoriasis has a major secondary impact on the lives of family members and partners. Br J Dermatol 2007 Jun;156(6):1245-1250.

9. Sampogna F, Abeni D, Gieler U, Tomas-Aragones L, Lien L, Titeca G, et al. Impairment of Sexual Life in 3,485 Dermatological Outpatients From a Multicentre Study in 13 European Countries. Acta Derm Venereol 2017 Apr 06,;97(4):478-482

10. Reich A, Hrehorów E, Szepietowski JC. Pruritus is an important factor negatively influencing the well-being of psoriatic patients. Acta Derm Venereol 2010 May;90(3):257-263.

11. First acne education program for Canadian high school students now available; shows positive improvements in teens after one month. Available at: https://www2.gov.bc.ca/gov/content/ health/keeping-bc-healthy-safe/pses-mpes/tanning-beds. Accessed June 2, 2019.

12. Jang $\mathrm{YH}$, Lee JS, Kim SL, Song CH, Jung HD, Shin DH, et al. A Family-Engaged Educational Program for Atopic Dermatitis: A Seven-Year, Multicenter Experience in Daegu-Gyeongbuk, South Korea. Annals of Dermatology 2015 Aug 1,;27(4):383388.

13. Grossman SK, Schut C, Kupfer J, Valdes-Rodriguez R, Gieler $\mathrm{U}$, Yosipovitch G. Experiences with the first eczema school in the united states. Clin Dermatol. 2018;36(5):662-667. Accessed May 29, 2019. doi: 10.1016/j.clindermatol.2018.05.006. 